\title{
Off Label Use of Exparel in an Axillary Block for Prolonged Postoperative Analgesia
}

\author{
Reet Lawhon, Robert LaCivita, John Fanouse, Dennis Feierman \\ Department of Anesthesiology, Maimonides Medical Center, Brooklyn, NY, USA \\ Email: dfeierman@maimonidesmed.org
}

Received 12 June 2015; accepted 19 July 2015; published 22 July 2015

Copyright (C) 2015 by authors and Scientific Research Publishing Inc.

This work is licensed under the Creative Commons Attribution International License (CC BY). http://creativecommons.org/licenses/by/4.0/

(c) (i) Open Access

\begin{abstract}
Post-operative analgesia is both necessary for patient comfort and satisfaction. Peripheral nerve block offer both good post-operative analgesia and patient satisfaction, but rarely cover the entire period of post-operative pain. Nonnarcotic drugs are extremely helpful, but with severe pain, narcotics are usually used to help with post-operative pain when the nerve block wears off. However, nausea and vomiting, as well as other side effects associated with narcotic use can be severe and limit their use for post-operative analgesia. The addition of supplements to the local anesthetic used for the nerve blocks can extend the analgesia, but few blocks last greater than 24 hours. We describe the use of a relatively new extended release bupivacaine, Exparel that provide significantly longer analgesia than presently used single shot techniques protocols for extended nerve block analgesia.
\end{abstract}

\section{Keywords}

Ambulatory, Nerve Block, Exparel

\section{Introduction}

Nausea and vomiting associated with narcotic use can be severe and limit their use for postoperative analgesia. Although there are non-opioid analgesics, these are usually inadequate, especially with large or invasive operations or painful procedures. For many orthopedic procedures, regional anesthesia may provide significant post-operative analgesia for several hours. The addition of supplements to the local anesthetics can extend the analgesia, but few blocks last greater than 24 hours and that still might be inadequate. We describe the use of a relatively new extended release bupivacaine, Exparel that provided significantly longer analgesia than presently used protocols. 


\section{Case Report}

A 62 year old Hispanic female presented for open reduction and internal fixation of a left distal radius fracture. She had no significant past medical history: the patient denied any cardiac, respiratory, endocrine, liver or kidney disorders. She denied the use of daily medications; however, during the preoperative interview, the patient described no drug allergies, but intolerance to narcotics causing the patient incapacitating nausea and vomiting. Her neurologic exam was grossly normal and the rest of her physical exam was benign. Preoperatively, we discussed with the patient and the surgeon the off label use of Exparel. The potential benefit of using the long acting bupivacaine was to minimize the use of narcotic postoperative, secondary to her intolerance of narcotics. Since other liposomal drugs, i.e., DepoDur, liposomal morphine, have been used both epidural and intrathecal spaces, we did not think the use of liposomal bupivacaine would cause neuronal toxicity. However, there would be a risk of a prolonged block.

\subsection{Preoperative}

Exparel is used for infiltration in the incision site after the operation for prolonged analgesia. Using an estimate of 72 hours for the complete release of the bupivacaine from the liposomes, if half the vial $(10 \mathrm{ml}$ of Exparel contains 133 mgs of bupivacaine) was used for the axillary block, each nerve bundle would receive less than 12 $\mathrm{mg} /$ day of bupivacaine. Therefore, it was decided to use the half vial of Exparel for the axillary block and the other half for infiltration of the incision. After application of standard ASA monitors, the patient was sedated with $2 \mathrm{mg}$ Midazolam and skin was prepped with chlorhexidine. An axillary block was performed using $10 \mathrm{ml}$ of Exparel expanded with $20 \mathrm{ml}$ normal saline ( $30 \mathrm{ml}$ total). Axillary artery and nerves were identified utilizing ultrasound. Using an out of place technique after disappearance of twitches at $0.4 \mathrm{~mA}, 8 \mathrm{ml}$ of diluted Exparel solution was injected at each nerve site (medial, ulnar and radial) and periarterial spread was observed. The remaining 6 cc was used to infiltrate musculocutaneous nerve in corachobrachialis muscle. Intercostobrachial nerve was blocked with $3 \mathrm{ml}$ of $0.25 \%$ bupivacaine for tourniquet pain. The patient was evaluated for sensory and motor blockade 30 minutes after block performed and had a dense sensory blockade and moderate motor block.

\subsection{Intraoperative}

The patient was taken to operating room. Anesthesia was induced with $2 \mathrm{mg} / \mathrm{kg}$ propofol, and the airway was maintained with a 3 laryngeal mask airway. Maintenance of anesthesia was achieved with $1 \%$ sevoflurane and an infusion of propofol at $75 \mathrm{mcg} / \mathrm{kg} / \mathrm{min}$. The surgery was performed uneventfully and an open reduction internal fixation distal radius was performed. Prior to closing surgeon expanded the last 10 cc of Exparel with 10cc of normal saline, infiltrated throughout surgical field including the periosteum, deep and superficial subcutaneous tissues. The wound was then closed and site immobilized by surgical team. The LMA was removed deep and patient emerged from anesthesia uneventfully, she reported zero pain and was transferred to the post anesthesia care unit (PACU).

\subsection{Postoperative Course}

Patient had no pain in the PACU, did not receive any narcotic in the PACU and was discharged home. Additionally, the patient did not complain of nausea or have postoperative vomiting. She was contacted daily for 3 days following procedure to assess the performance of the block. Due to immobilization of the arm it was difficult to assess recovery from motor blockade, but a sensory block lasted 56 hours. At this point, the patient experienced 2/10 pain, and took 1 tab of Percocet 5/325. The patient complained of dysphoria after the narcotics and subsequently took no further analgesics.

\section{Discussion}

The use of regional and anesthesia and reduced narcotics has been shown to provide superior same-day recovery compared to general anesthesia [1]. Our overall objective would be to reduce or eliminate the use narcotic for postoperative pain. Exparel, liposomal bupivacaine is a sustained release bupivacaine. Its effects have been noted to last up to 72 hrs [2]. This case highlights the successful use of Exparel for postoperative pain relief in a 
Table 1. Risk factors for difficult postoperative pain management.

Obesity
COPD
Preoperative Chronic Pain
History of Narcotic Aabuse
History of Alcohol Use/Abuse
Significant History of Nausea/Vomiting with Narcotics
Stroke with Significant Residual
Dementia
Frail Elderly Patients
Extremely Difficult Airway

brachial plexus nerve block. This has the potential for use in patients with contraindications to narcotic use and to minimize the side effects of narcotics. Avoiding narcotics can decrease incidence of post-operative nausea and vomiting. Furthermore, with decreased pain, nausea and vomiting there can be a shortened PACU stay, reducing costs and improving patient satisfaction. With increasing rates of obesity, Exparel can potentially minimize narcotic use which may increase the safety of post-operative analgesia. Alternate strategies for postoperative pain relief in ambulatory surgery include indwelling catheters to provide slow infusions of local anesthetics. With the single shot technique with Exparel, you have the benefit of prolonged pain relief without the risk of infection and/or catheter dislodgement.

The use of liposomal bupivacaine significantly reduced the use of post-operative narcotics in this patient. We believe that the use of extended release local anesthetics should be considered when there is a need to reduce the use of post-operative narcotics or postoperative pain management could be very difficult (Table 1).

\section{Conclusion}

This case highlights the successful use of Exparel for extended post-operative pain relief in a brachial plexus nerve block. This has the potential for use in patients to minimize their use of narcotics. This case suggests that the use of Exparel in a brachial plexus block may reduce the use of post-op narcotics, and other post-operative complications, e.g., nausea and vomiting related to the use of opioids.

\section{Disclousure}

Reet Lawhon is a consultant and a speaker for Pacira Pharmaceuticals.

\section{References}

[1] Hadzic, A., Williams, B.A., Karaca, P.E., Hobeika, P., Unis, G., Dermksian, J., Yufa, M., Thys, D.M. and Santos, A.C. (2005) For Outpatient Rotator Cuff Surgery, Nerve Block Anesthesia Provides Superior Same-Day Recovery over General Anesthesia. Anesthesiology, 102, 1001-1007. http://dx.doi.org/10.1097/00000542-200505000-00020

[2] Gorfine, S.R., Onel, E., Patou, G. and Krivokapic, Z.V. (2011) Bupivacaine Extended-Release Liposome Injection for Prolonged Postsurgical Analgesia in Patients Undergoing Hemorrhoidectomy: A Multicenter, Randomized, DoubleBlind, Placebo-Controlled Trial. Diseases of the Colon and Rectum, 54, 1552-1559.

http://dx.doi.org/10.1097/DCR.0b013e318232d4c1 